\title{
USO DEL ESTÁNDAR LTI PARA INTEGRAR SISTEMAS DE GESTIÓN DE APRENDIZAJE Y LABORATORIOS ONLINE
}

\author{
I. Ruano Ruano, E. Estévez Estévez, J. Gómez Ortega y J. Gámez García \\ Grupo de Robótica, Automática y Visión por Computador \\ Universidad de Jaén, Campus Las Lagunillas s/n, ES-23071, Jaén (España) \\ alonso@ujaen.es
}

\begin{abstract}
Resumen
La importancia del trabajo práctico es innegable en la mayoría de los estudios relacionados con las ciencias, tecnologías, ingenierías y matemáticas (en inglés Science, Technology, Engineering and Mathematics o estudios STEM). Los laboratorios online permiten que los estudiantes realicen este tipo de trabajo a través de Internet. Sin embargo, Los Sistemas de Gestión de Aprendizaje (LMS) constituyen el entorno de trabajo natural de los estudiantes en Internet, por ello se debe lograr la integración de LMS y laboratorios online. Este trabajo presenta el estándar Learning Technology Interoperability, más conocido como LTI, para lograr la integración de LMS y Laboratorios Online. Muestra su funcionamiento, la situación actual de LTI, las versiones de LTI existentes y las diferentes maneras en las que se puede utilizar para lograr una integración. Adicionalmente se incluye un ejemplo de integración LTI de un laboratorio online en un LMS $y$ las ventajas que se pueden obtener de esta integración.
\end{abstract}

Palabras clave: Laboratorios Online, Sistemas de Gestión de Aprendizaje (LMS), Learning Technology Interoperability (LTI), Integración de sistemas, Experimentos.

\section{INTRODUCCIÓN}

En los estudios STEM (ciencias, tecnologías, ingenierías y matemáticas) el trabajo de prácticas es muy importante [5]. Con la llegada de la educación a través de Internet o e-learning aparecieron los laboratorios online [20], [7] y tras muchos estudios se ha producido un acuerdo sobre los beneficios que aportan en la enseñanza, principalmente en las áreas de ciencias e ingenierías [12].

Sin embargo, el mayor exponente del e-learning en la educación son los Sistemas de Gestión de aprendizaje o LMS (Learning Management System). Los LMS también son conocidos por el nombre de entornos de aprendizaje virtual (Virtual Learning Environment, VLE) o plataformas de docencia virtual y hoy día están plenamente instalados en las instituciones educativas universitarias. Ya que el entorno de trabajo natural de los estudiantes en e-learning es un LMS, es lógico que ya en el año 2006 la integración LMS-Lab fuera señalada como un objetivo a conseguir para poder tratar los laboratorios online como objetos de aprendizaje definidos junto al resto de recursos de aprendizaje del LMS y no aislados [13]. Desde entonces se han realizado múltiples trabajos con el objetivo de lograr esta integración, algunos de ellos se han basado en el desarrollo de propuestas tecnológicas ad hoc [31], [32] mientras que otros han tratado de lograrlo mediante el uso de estándares como SCORM (Shareable Content Object Reference Model) [1], [24], [27] o LTI (Learning Technology Interoperability) [6], [10], [23].

En el ámbito tecnológico, el objetivo de un estándar es establecer normas que permitan el entendimiento y la colaboración entre sistemas. Su uso proporciona una serie de ventajas muy importantes [1]. Este trabajo está enfocado en el estándar LTI [30] y su uso en laboratorios online y LMSs para lograr su integración. Gracias a LTI un laboratorio online, localizado en un servidor externo al LMS, puede ser utilizado por los estudiantes accediendo al mismo tras identificarse en el LMS y abrir un elemento del enlazado con la aplicación del laboratorio. El mismo LTI puede servir para que un software de laboratorio disponible online pueda incluir y presentar recursos de un LMS (externo al laboratorio) que se pueden presentar a los estudiantes junto a las prácticas.

En las próximas secciones se va a mostrar algunas generalidades del estándar LTI (Apartado 2) incluyendo su funcionamiento, versiones existentes y el uso del mismo en el mercado de software. En el apartado 3 se mostrarán esquemas de integración Lab-LMS que se pueden lograr gracias a LTI mientras que el apartado 4 mostrará un ejemplo de integración y las ventajas que puede aportar. Para terminar, el apartado 5 incluye las conclusiones principales de este trabajo.

\section{EL ESTÁNDAR LTI}

LTI es un estándar desarrollado por IMS Global Learning Consortium [15], se trata de una institución sin ánimo de lucro formada por miembros cuya 
misión principal dice ser “...avanzar en tecnología que pueda escalar y mejorar de manera asequible la participación y el logro educativo" (extraído de su web). Para ello se dedica al desarrollo y mantenimiento de estándares de interoperabilidad abiertos, apoya y fomenta su adopción con servicios técnicos y programas que resaltan las prácticas efectivas. En el informe anual del año 2018 [17] se indica que el número de miembros de IMS son 490, el número de certificaciones realizadas en el año fueron 523, lo que supuso un aumento del $15 \%$ de los ingresos y también se incluye que existe un amplio uso en el mundo de los estándares IMS.

El estándar LTI se usa para enlazar herramientas externas (contenidos y recursos) con plataformas de aprendizaje. Los laboratorios online constituyen sólo uno de los tipos de recursos que son susceptibles de ser enlazados con estas plataformas. Aunque si se amplía la visión del laboratorio online, y se le considera como una plataforma de aprendizaje en sí mismo, también existe la posibilidad de usar LTI para enlazar otras herramientas externas al laboratorio con el mismo.

\subsection{FUNCIONAMIENTO DE LTI}

LTI permite la integración entre aplicaciones de aprendizaje enriquecidas (creadas por cualquier método y alojadas remotamente) con plataformas de aprendizaje como LMSs, portales Web, repositorios de objetos de aprendizaje o cualquier otro entorno de aprendizaje. La terminología LTI llama herramientas (tools) a las aplicaciones de aprendizaje creadas por tool providers (Proveedores de herramientas) y tool consumers (Consumidores de herramientas) a los LMSs, plataformas o portales Web. La Figura 1 muestra el esquema de un ejemplo de uso.

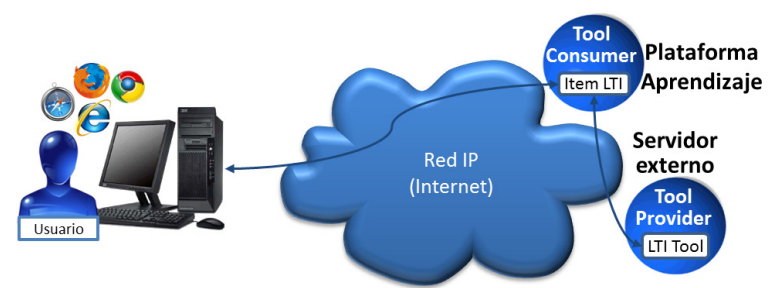

Figura 1: Ejemplo de uso de LTI

Estas especificaciones se utilizan más en el ámbito académico que otras áreas. El estándar permite integraciones de tipo "plug and play" entre un LMS y aplicaciones externas (p.e. un laboratorio) de forma fácil, barata y rápida. Para ello el LMS y la aplicación deben ser compatibles con LTI. Otros ejemplos de herramientas, aparte de los laboratorios online, que podrían ser utilizadas son: e-portafolios, libro electrónico, editor de documentos o presentaciones, herramientas de colaboración, recursos de biblioteca, herramientas de evaluación, herramientas de tareas, herramientas de clasificación, herramientas de video o herramientas de temas específicos. Usando LTI, un usuario del LMS podría entrar en el LMS y ejecutar un laboratorio externo, para ello, el laboratorio debe usar la API LTI para comunicarse con el LMS. En este caso, las comunicaciones que se puede establecer entre el LMS y el laboratorio debe restringirse a las establecidas por el estándar LTI utilizado, entre ellas se encuentran las necesarias para que el consumidor de herramientas (LMS normalmente) envíe al proveedor de herramientas los datos de Lanzamiento básicos (Basic Launch Data). Entre otras facilidades, estos datos van a permitir que la identificación del usuario que utiliza el LMS pueda ser utilizada en la herramienta lanzada (laboratorio online), también es posible trasladar del LMS al laboratorio otros datos como el entorno en el que se ejecuta el laboratorio en el LMS (p.e. curso), el rol o permisos del usuario en el mismo, otros que identifican al LMS, la instancia ejecutada y otras características del LMS, hay otros datos que especifican características del interfaz en el que se va a ejecutar el software del laboratorio (anchura y/o altura entre otros) y finalmente datos referidos a los resultados anteriores obtenidos por el usuario en el laboratorio. Por otro lado, el laboratorio podría registrar el resultado de las acciones que realiza el usuario directamente en el LMS.

\subsection{EXTENSIÓN DE LTI Y VERSIONES}

LTI está bastante extendido, existen muchos productos (aplicaciones y plataformas de aprendizaje) que lo utilizan, tanto plataformas de aprendizaje que suelen actuar como consumidoras de herramientas LTI (tool consumers) como plataformas que ofrecen aplicaciones que pueden ser consumidas (tool providers). En la web de IMS Global existe un listado oficial de productos que han superado la certificación de IMS Global para LTI con el que se puede ofrecer una visión del uso que se hace (https://site.imsglobal.org/certifications). En la misma se puede ver como el número de productos que han superado alguna de las certificaciones LTI oficiales es 275 (10-06-2019). La última actualización de LTI (mayo de 2019) lo constituye LTI advantange, que es un paquete de tres servicios esenciales para usuarios finales que se basan en LTI v1.3 por lo que se puede considerar un estándar plenamente activo y actualizado actualmente. Existen numerosas versiones de LTI, aunque no todas las versiones están igual de extendidas, como muestra el siguiente resumen:

- LTI versión 1.0: 250 productos

- LTI versión 1.1: 96 productos

- LTI versión 1.1.1: 20 productos

- LTI versión 1.2: 21 productos

- LTI versión 1.3: 17 productos

- LTI versión 2.0: 6 productos

- LTI Advantage: 17 productos 
La hoja de ruta de adopción de LTI recomendada por IMS aconseja pasar directamente de la versión $1.1 \mathrm{a}$ la 1.3 olvidándose de las versiones 1.1.1, 1.2 y 2.0. El uso del estándar LTI es aún mayor que el que pueda parecer al ver estos números, ya que existen otros productos que soportan LTI, pero que no han obtenido la certificación de IMS Global (Por ejemplo el LMS ILIAS [14]). Esto se debe a que al tratarse de un estándar abierto no es obligatorio el superar una certificación para adoptarlo. La certificación simplemente garantiza que los productos enumerados en el directorio cumplen con los estándares de IMS para los cuales han pasado las pruebas.

Todas las plataformas de docencia virtual o LMS más utilizadas en el mercado tienen versiones que soportan al menos una versión de LTI (Canvas [33], Blackboard Learn [3], Moodle [8], Brightspace D2L [4], Google G-Suite [11], Sakai [29]). Aunque hay que tener en cuenta la versión utilizada, por ejemplo Moodle v3.7 cumple sólo la certificación LTIv1.3 y LTI Advantage de IMS Global mientras que Moodle 3.3.1+ posee las certificaciones LTI v1.0, LTI v1.1 y LTI v2.0.

\section{LTI PARA INTEGRAR LMSS Y LABORATORIOS ONLINE}

La integración de un LMS y un Laboratorio online (también llamado WebLab) puede hacerse de distintas formas que se van a enumeran y explicar brevemente en los siguientes subapartados.

\subsection{NATIVA: USO DESDE UN LMS DE UN WEBLAB EXTERNO}

Esta es la forma más natural y más fácil de imaginar la integración de un LMS y un laboratorio. La Figura 2 muestra el esquema el esquema más simple de este caso, en el que un usuario se conecta a un LMS en el que se encuentra un elemento LTI que al ser activado lanza un laboratorio virtual ubicado en un servidor externo que el usuario ejecuta en su pantalla. Si el lanzamiento del software del laboratorio que realiza el LMS se produce embebido en el entorno del LMS, el usuario obtiene la sensación de que el laboratorio está plenamente integrado en el LMS.

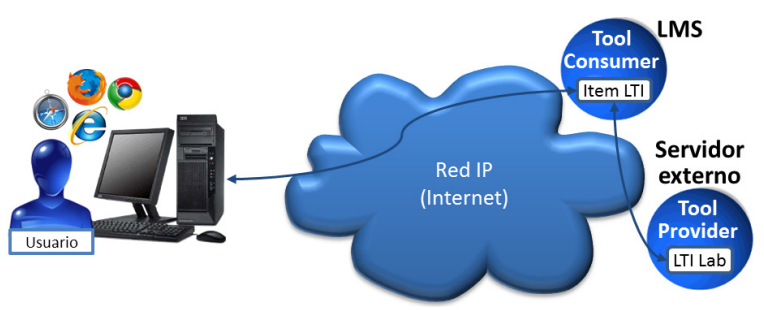

Figura 2: WebLab virtual integrado en LMS con LTI

El esquema mostrado en la figura 2 se puede volver más complejo; por ejemplo, en el caso de que el laboratorio online sea remoto, será necesario que el software del laboratorio LTI también tenga acceso a un sistema real, que podría estar en el mismo servidor externo o conectado a otro servidor, como muestra el ejemplo de la Figura 3.

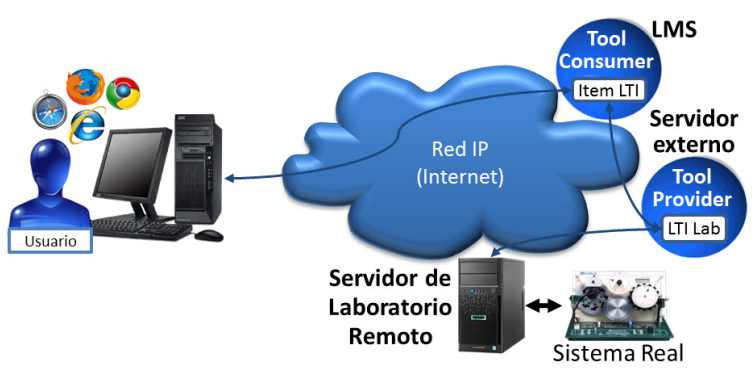

Figura 3: WebLab remoto integrado en LMS con LTI

\subsection{INVERSA: USO DESDE UN WEBLAB DE RECURSOS DEL LMS}

Esta forma es algo menos intuitiva, pero no por ello deja de ser una manera de integrar un laboratorio online y un LMS. En este caso el laboratorio actúa como plataforma de aprendizaje que lanza una herramienta externa gracias al estándar LTI. Este caso puede ser útil para añadir al software de un laboratorio cualquier recurso existente en un LMS que sea compatible con LTI y pueda operar como un proveedor de herramientas (tool provider).

Existen LMS que pueden ofrecer estos servicios de forma nativa [22] o gracias a algún software añadido o plugin [19]. Ejemplos de recursos puede ser un foro, un test, un contenido, una encuesta. Por ejemplo, se podría tener un laboratorio online que por LTI muestre al alumno un contenido del LMS antes de realizar los experimentos, tras acabar la experimentación presente un test compatible LTI ubicado en el LMS para valorar los conocimientos adquiridos y que incluya, también por LTI, una encuesta también ubicada en el LMS para conocer la opinión del estudiante sobre su experiencia en el laboratorio. En este caso el LMS se comporta como un proveedor de herramientas (tool provider) como muestra la integración ejemplo de la Figura 4.

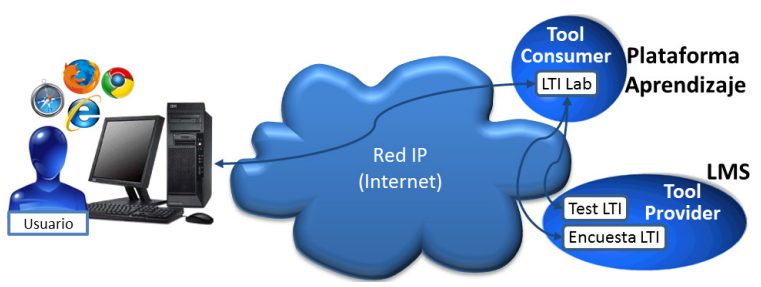

Figura 4: Recursos LMS integrados en WebLab con LTI

\subsection{MIXTA: USO DESDE UN LMS DE UN WEBLAB EXTERNO QUE CONTIENE HERRAMIENTAS DEL LMS}

Este último caso consiste en una combinación de los dos anteriores, el alumno se identifica en un LMS en 
el que puede lanzar un laboratorio online que se encuentra localizado en un servidor externo e incluye recursos de un LMS (que puede ser el mismo u otro, como el ejemplo mostrado en la Figura 5).

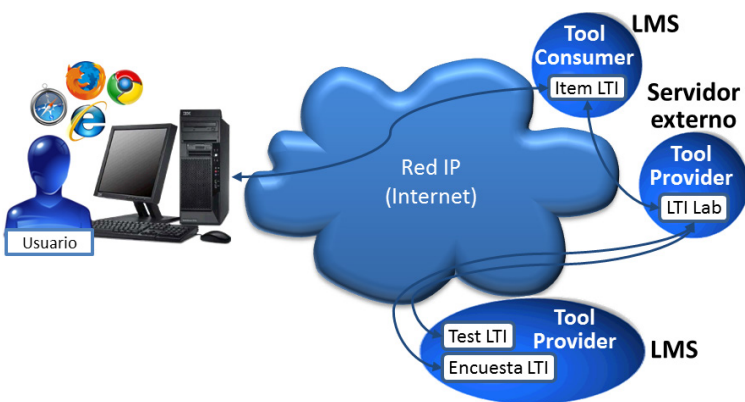

Figura 5: WebLab virtual integrado en LMS con LTI consumiendo recursos de otro LMS integrados también con LTI

\section{EJEMPLO DE INTEGRACIÓN LAB-LMS}

Se va a describir un ejemplo de integración LMS-Lab basada en LTI de tipo nativa, es decir, la descrita en el subapartado 3.1. Este ejemplo está accesible en: https://ev.ujaen.es/goto formacion_xxco 31387.html

\subsection{EL LMS}

El LMS utilizado ha sido la plataforma ILIAS de evaluación de docencia virtual de la Universidad de Jaén. Esta plataforma ILIAS tiene instalado el software "External Content plugin for ILIAS" [21] que permite integrar contenido externo o herramientas como objetos del repositorio. Este plugin soporta la especificación LTI 1.1 y permite la definición flexible de otros interfaces. Gracias a este añadido se pueden crear recursos de tipo "Contenido Externo" que enlazan con herramientas externas al LMS. En la Figura 6 se muestra una captura parcial de la ventana de configuración de uno de estos recursos.

Para poder integrar un laboratorio externo en el LMS es necesario configurar una serie de campos que indiquen al LMS la dirección en la que se encuentra el software del laboratorio que se quiere enlazar y unos parámetros de seguridad. En la Figura 6 se ha ocultado la dirección de enlace del laboratorio así como la clave de lanzamiento y la palabra secreta compartida que el proveedor de herramientas, los dos últimos parámetros aportan seguridad evitando que cualquier LMS (tool consumer) pueda consumir los servicios del laboratorio online. El profesor o tutor que desee integrar el laboratorio para sus alumnos en el LMS deberá conocer estos parámetros $\mathrm{y}$ configurarlos correctamente. En la misma figura también existen otros parámetros que permiten decidir la forma en la que se quiere ejecutar el laboratorio, indicando dimensiones del contenedor en el que se va a mostrar y si se hará embebido en el mismo entorno del LMS, en otra pestaña del navegador o en otra ventana de navegador. Si el LMS en el que se trate de realizar la integración es de otro tipo distinto a ILIAS tendrá otras pantallas de configuración distintas, pero los parámetros deberán ser similares.

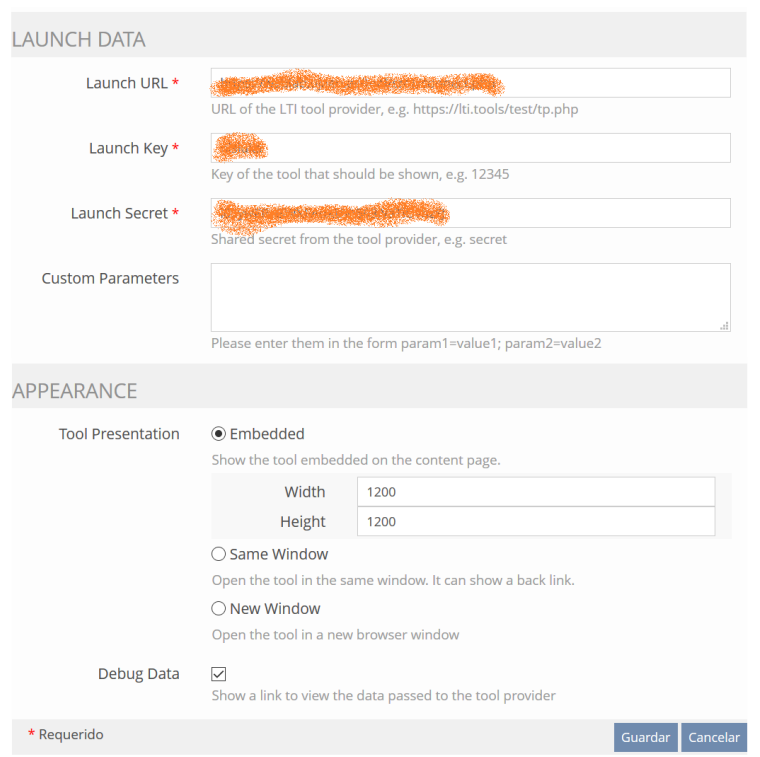

Figura 6: Configuración de contenido externo en LMS

\subsection{EL LABORATORIO ONLINE}

El software del laboratorio utilizado se desarrolló en lenguaje JavaScript utilizando EjJS [9], el objetivo del mismo es el control del movimiento (posición/velocidad angular) de un motor de corriente continua mediante el ajuste de un controlador PID (Proporcional, Integral Derivativo), muy útil para los estudiantes de grados de ingeniería. La Figura 7 muestra el interfaz de usuario del laboratorio.

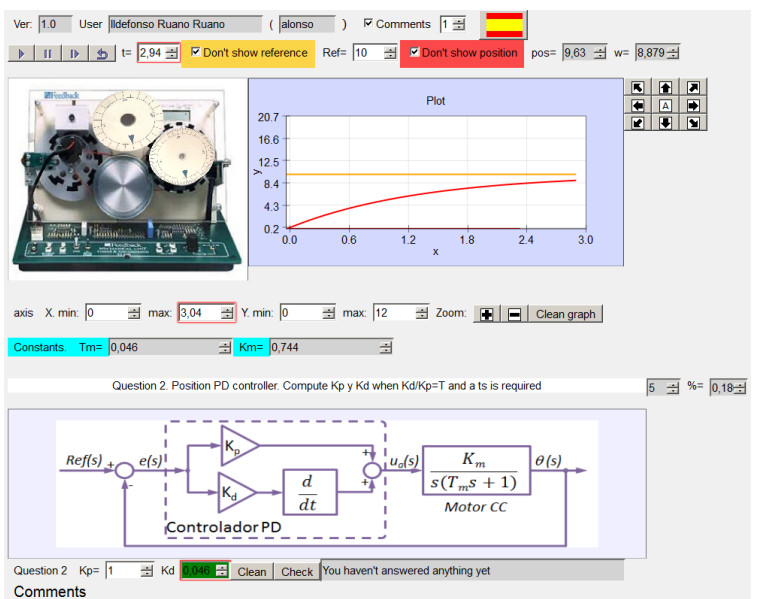

Figura 7: Interfaz de usuario del laboratorio ejemplo 
Una versión anterior de este laboratorio ya fue utilizada para lograr una integración avanzada con un LMS, aunque en este caso el laboratorio se encontraba en el mismo LMS y el estándar utilizado para lograr la integración fue SCORM [26]. Desde hace bastante tiempo, las aplicaciones desarrolladas con EJjS se pueden integrar fácilmente en un LMS de tipo Moodle utilizando el plugin utilizado a dicho fin [31]. Por otro lado, las últimas versiones del programa EJjS ofrecen una opción de exportación al formato SCORM de las aplicaciones desarrolladas (simulaciones o software de laboratorio). De este modo, se obtienen paquetes SCORM que contienen en laboratorio deseado. Esto permite la integración inmediata de los laboratorios desarrollados en EJjS en cualquier LMS compatible con el estándar SCORM [25] al incluir el paquete SCORM generado por EJjS en el LMS.

La opción aquí presentada constituye una nueva opción de integración. Para poder cumplir con el estándar LTI, se utilizó la librería PHP de proveedor de herramientas LTI (LTI tool Provider library PHP) [16] y el código ejemplo PHP de proveedor de herramientas LTI (LTI Sample Tool Provider PHP) [18], ambos facilitados libremente por IMS. El código ejemplo del proveedor de herramientas LTI se modificó para sustituir la funcionalidad original por las funciones JavaScript del laboratorio creado con EJsS. Los códigos resultantes se ubicaron en un servidor Linux en el que se tiene un servidor Web Apache, PHP y una base de datos MySQL debidamente configurado con los parámetros necesarios para dar de alta el LMS ILIAS y permitir, de este modo, que los estudiantes puedan lanzar la aplicación del laboratorio desde el LMS.

\subsection{VENTAJAS DE LA INTEGRACIÓN}

Se van a enumerar las ventajas que se pueden obtener en el ejemplo de integración LMS-Lab descrito en párrafos anteriores. El principal y más importante es que el estudiante percibe el laboratorio como otro objeto docente del LMS, aunque no esté ubicado físicamente en él, por lo que la ejecución del laboratorio se realiza en un entorno conocido por el alumno. Además, según las posibilidades del LMS, se podrá presentar junto a otros recursos para que el alumno no perciba al laboratorio como un recurso aislado. Otro grupo de ventajas vienen dadas por la identificación del alumno que el LMS puede trasladar al laboratorio como datos de lanzamiento. Esto permite la posibilidad de que se puedan programar laboratorios con estas características:

- Ejecución personalizada a gusto del estudiante (volumen, subtítulos, idioma de interfaz, etc.)

- Ejecución de prácticas personalizadas según necesidades del alumno o trayectoria previa.
- Seguimiento del trabajo práctico realizado por parte de alumnos y tutores.

- Almacenamiento de logros, calificaciones parciales $y$ totales en el servidor del laboratorio.

- Almacenamiento en el LMS del resultado final obtenido

Además, se pueden programar secuencias de aprendizaje en el LMS que permitan establecer una serie de objetos y recursos docentes (entre los que se encuentre el laboratorio LTI) que el alumno debe superar y que, en función de los resultados obtenidos, pueda cambiar. Finalmente, otra ventaja adicional que presenta este tipo de integración de un laboratorio basada en LTI respecto a la que se puede obtener con el estándar SCORM [26] es que una única aplicación del laboratorio instalada en un servidor externo puede ser utilizada simultáneamente por múltiples LMS, lo que le hace más adecuado cuando se quiere compartir recursos tipo laboratorios online entre distintas organizaciones.

\section{CONCLUSIONES}

Este trabajo ha mostrado una breve introducción al estándar LTI como tecnología clave para integrar un laboratorio online y un LMS. Se han perfilado las distintas posibilidades de integración que se pueden producir:

- Nativa. Integración, como un recurso más de un LMS, de un laboratorio online docente localizado en un servidor externo al LMS.

- Inversa. Integración de recursos de aprendizaje de un LMS en un laboratorio online accesible directamente.

- Mixta. Integración, como un recurso más de un LMS, de un laboratorio online docente localizado en un servidor externo que incluye recursos de aprendizaje de un LMS que puede ser el mismo u otro.

El trabajo también incluye un ejemplo de integración nativa avanzada [28] basada en LTI que ayuda a entender como se debe realizar la creación de un laboratorio con estas características, que acciones se deben realizar en el LMS y las ventajas que se pueden obtener con este tipo de integración.

\section{Agradecimientos}

Este trabajo ha sido parcialmente subvencionado por los proyectos DPI2016-78290-R y PID70_2016-17.

\section{English summary}

USE OF THE LTI STANDARD TO ACCESSS ONLINE LABORATORIES FROM LEARNING MANAGEMENT SYSTEMS 


\begin{abstract}
The importance of practical work is undeniable in most studies related to science, technology, engineering and mathematics (STEM). Online labs allow students to do this type of work through the Internet. However, the Learning Management Systems (LMS) constitute the natural work environment of students on the Internet, which is why the integration of LMS and online laboratories must be achieved. This work presents the Learning Technology Interoperability standard, better known as LTI, to achieve the integration of LMS and Online Laboratories. It shows how it works, current LTI situation, existing LTI versions, and different ways in which it can be used to achieve integration. Additionally, an example of integration of an online laboratory in an LMS is included and the advantages that can be obtained from this integration.
\end{abstract}

Keywords: Online Laboratories, Learning Management System (LMS), Learning Technology Interoperability (LTI), System Integration, Experiments.

\section{Referencias}

[1] Bellido, L., Mateos, V., Villagrá, V. A., Fernández, D., and Walid, O., "Remote access to computer networking laboratories," 2012 9th Int. Conf. Remote Eng. Virtual Instrumentation, REV 2012, 2012.

[2] Berlanga Reyes, A., Fernández Manjón, B., Martínez Ortiz, I., Moreno Ger, P., and Sierra Rodríguez, J. L., "Estándares en e-learning y diseño educativo" 2011.

[3] Blackboard, "Blackboard Learn Web Site" [Online]. Available: https://www.blackboard.com/blackboardlearn/index.html. [Accessed: 21-Jun-2019].

[4] Brightspace, "Desire2Learn (D2L) Web Site" [Online]. Available: https://www.d2l.com/es/. [Accessed: 21-Jun-2019].

[5] Clough, M. P., "Using the laboratory to enhance student learning" Learn. Sci. Sci. Learn., no. JANUARY 2002, pp. 85-94, 2002.

[6] Colbran, S., Schulz, M., "An Update to the Software Architecture of the iLab Service Broker", Proceedings of 2015 12th International Conference on Remote Engineering and Virtual Instrumentation (REV), pp. 90-93, Feb. 2015.

[7] Corter, J. E., Nickerson, J. V., Esche, S. K., Chassapis, C., Im, S., and Ma, J., "Constructing reality: A Study of Remote, Hands-On, and Simulated Laboratories" ACM Trans. Comput. Interact., vol. 14, no. 2, pp. 7-es, Aug. 2007.
[8] Dougiamas, M. and Moodle-Community, "Moodle Web Site" 2002. [Online]. Available: https://moodle.org/. [Accessed: 21-Jun-2019].

[9] Esquembre, F., "Easy Java Simulations: a software tool to create scientific simulations in Java" Comput. Phys. Commun., vol. 156, no. 2, pp. 199-204, Jan. 2004.

[10] Garcia Clemente, F. J. et al, "Collecting Experience Data from Remotely Hosted Learning Applications", Online Engineering \& Internet of Things, pp. 170-181, 2018.

[11] Google, "Google G-Suite Web Site." [Online]. Available:

https://edu.google.com/products/gsuite-foreducation/. [Accessed: 21-Jun-2019].

[12] Gravier, C., Fayolle, J., Bayard, B., Ates, M., and Lardon, J., "State of the Art About Remote Laboratories Paradigms - Foundations of Ongoing Mutations" Int. J. Online Eng., vol. 4, no. 1, pp. 19-25, 2008.

[13] Gravier, C., Fayolle, J., Noyel, G., Lelevé, A., and Benmohamed, H., "Distance learning: Closing the gap between remote labs and learning management systems" 2006 1st IEEE Int. Conf. E-Learning Ind. Electron. ICELIE, pp. 130-134, 2006.

[14] ILIAS, "Integriertes Lern-,Informations- und Arbeitskooperations-System (ILIAS)" [Online]. Available: http://www.ilias.de. [Accessed: 28Jul-2016].

[15] IMS Global Learning Consortium (IMS), "IMS Global Learning Consortium (IMS)" [Online]. Available: https://www.imsglobal.org/. [Accessed: 21-Jun-2019].

[16] IMS Global Learning Consortium (IMS), "LTI Tool provider Library PHP" 2016. [Online]. Available: https://github.com/IMSGlobal/LTITool-Provider-Library-PHP/wiki. [Accessed: 07-Aug-2018].

[17] IMS Global Learning Consortium, "Creating the Future EdTech Ecosystem Together. 2018 annual report. Fiscal results and progress" 2018.

[18] IMS Global Learning Consortium, "LTI Sample Tool Provider PHP” 2016. [Online]. Available: https://github.com/IMSGlobal/LTI-SampleTool-Provider-PHP/wiki. [Accessed: 07-08-18].

[19] Leyva, J., "Plugin LTI Provider de Moodle" [Online]. Available: https://moodle.org/plugins/local_tiprovider. [Accessed: 21-Jun-2019].

[20] Ma J. and Nickerson, J. V., "Hands-on, simulated, and remote laboratories: A Comparative Literature Review" ACM Comput. Surv., vol. 38, no. 3, pp. 7-es, Sep. 2006.

[21] Neumann, F. and Copado, J., "Plugin External Content (with LTI v1.1 support) de ILIAS" 2018. [Online]. Available: https://docu.ilias.de/goto_docu_cat_3466.html. [Accessed: 21-Jun-2019]. 
[22] Neumann, F., "ILIAS as LTI Tool Provider" $2015 . \quad$ [Online]. Available: https://docu.ilias.de/goto_docu_wiki_wpage_37 75_1357.html. [Accessed: 21-Jun-2019].

[23] Orduna, P. et al., "An Extensible Architecture for the Integration of Remote and Virtual Laboratories in Public Learning Tools" IEEE Rev. Iberoam. Tecnol. del Aprendiz., vol. 10, no. 4, pp. 223-233, Nov. 2015.

[24] Ruano, I., Cano, P., Gámez, J., and Gómez, J., "Advanced LMS Integration of SCORM Web Laboratories" IEEE Access, vol. 4, pp. 63526363, 2016.

[25] Ruano, I., Estévez, E., Esquembre, F., and García, J., "Pautas para la integración portable de aplicaciones EjsS en Sistemas de Gestión de Aprendizaje," in XXXIX JORNADAS DE AUTOMÁTICA, 2018, pp. 135-144.

[26] Ruano-Ruano, I., Gámez García, J., and Gómez Ortega, J., "Laboratorio Web SCORM de Control PID con Integración Avanzada," Rev. Iberoam. Automática e Informática Ind. RIAI, vol. 13, no. 4, pp. 472-483, Oct. 2016.

[27] Ruano-Ruano, I., Gámez, J., Dormido, S. and Gómez, J., "A Methodology to Obtain Learning Effective Laboratories with Learning Management System Integration" IEEE Trans. Learn. Technol., vol. 9, no. 4, pp. 391-399, 2016.

[28] Ruano-Ruano, I., Gámez-García, J., and Gómez-Ortega, J., "Modos de integración de laboratorios online en sistemas de gestión de aprendizaje" in XXXVII Jornadas de Automática, 2016, pp. 653-660.
[29] Sakai, "Sakai Web Site." [Online]. Available: https://www.sakailms.org/.

[30] Severance, C., Hanss, T., and Hardin, J., "Ims learning tools interoperability: Enabling a mash-up approach to teaching and learning tools" Technol. Instr. Cogn. Learn., vol. 7, no. 3-4, pp. 245-262, 2010.

[31] Torre, L. de la, "New Generation Virtual and Remote Laboratories: Integration Into Web Environments 2.0 With Learning Management Systems" Spanish Open University (UNED), 2013.

[32] Trenas, M. A., Ramos, J., Gutiérrez, E. D., Romero, S., and Corbera, F., "Use of a new moodle module for improving the teaching of a basic course on computer architecture" IEEE Trans. Educ., vol. 54, no. 2, pp. 222-228, May 2011.

[33] Whitmer, B. and Daley, D., "Canvas Web Site" 2008. [Online]. Available: https://www.canvaslms.com/.

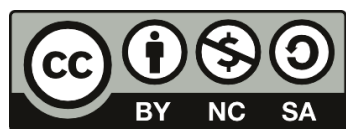

(C) 2019 by the authors. Submitted for possible open access publication under the terms and conditions of the Creative Commons Attribution CC BY-NC-SA 4.0 license (https://creativecommons.org/licenses/bync-sa/4.0/deed.es). 